\title{
Kinetics of human-T cell leukemia virus type 2 (HTLV-2) mRNA expression in PBMCs isolated from infected subjects Cecilia Bender ${ }^{1}$, Paola Ronzi ${ }^{3}$, Francesca Rende ${ }^{2}$, Alessia Cotena ${ }^{1}$, Marco Turci*1, Ilaria Cavallari², Vincenzo Ciminale ${ }^{2}$, Claudio Casoli $^{3}$ and Umberto Bertazzoni ${ }^{1}$
}

\author{
Address: ${ }^{1}$ Dept of Mother and Child, Biology and Genetics, Università degli Studi di Verona, Italy, ${ }^{2}$ Dept. of Oncology, Università degli Studi di \\ Padova, Italy and ${ }^{3}$ Dept of Clinical Sciences, Università degli Studi di Milano, Italy \\ * Corresponding author
}

\author{
from Frontiers of Retrovirology: Complex retroviruses, retroelements and their hosts \\ Montpellier, France. 21-23 September 2009 \\ Published: 24 September 2009 \\ Retrovirology 2009, 6(Suppl 2):P86 doi:I0.1 I86/I 742-4690-6-S2-P86
}

This abstract is available from: http://www.retrovirology.com/content/6/S2/P86

(C) 2009 Bender et al; licensee BioMed Central Ltd.

The analysis of HTLV-2 expression during the infection process is essential to understand the influence of viral gene products in proliferation, cell cycle and signalling. To date, very little information has been obtained on the profile and temporal regulation of HTLV-2 gene expression in infected cells.

To address this point, time course of transcription of individual HTLV-2 viral mRNAs was evaluated by real time RT-PCR using splice-junction-specific primers. PBMCs from HTLV-2B infected patients were cultured with IL-2 and the level of the different mRNAs analysed at the following time points: $0,2,4,8,21$ and 48 hours.

The results obtained showed an early transcription of tax/ rex regulatory transcript, followed by $p 28-p 22 / p 20$ rex-2 and by a gradual and steady increase of gag/pol structural transcripts and of $p 28-p 22 / p 20$ rex-1. The level of expression of $\mathrm{gag} / \mathrm{pol}$ and $p 28-p 22 / \mathrm{p} 20$ rex-2 was about $10^{3}-10^{4}$ fold higher than tax/rex and $p 28-p 22 / p 20$ rex-1. These findings indicate that the expression of different HTLV-2 genes follows a distinct timing in PBMCs isolated from infected patients. The regulatory transcripts are the first to be expressed whereas the structural mRNAs are expressed at a later time point. Moreover, the level of expression of the accessory genes was lower than that of structural viral genes.
This study identified distinct patterns of expression of HTLV-2 transcripts in infected PBMCs, thus indicating that the control of viral gene expression is highly regulated both in its kinetics and expression level.

Further studies are currently being performed to investigate the kinetics of gene expression of HTLV-2A subtype in transiently transfected human $\mathrm{T}$ cells. 